ICCM 2007 - Vol. II · 1-4

\title{
Cohomology algebras in symplectic, Kähler and algebraic geometry
}

\author{
Claire Voisin *
}

\begin{abstract}
We show a number of applications to geometry of the study of cohomology algebras of various kinds of manifolds. The main tool is Hodge theory, and we use it to show that projective complex manifolds are more restricted topologically than compact Kähler manifolds. We also make explicit numerous constraints satisfied by cohomology algebras of compact Kähler manifolds, making them very non generic amongst cohomology algebras of symplectic manifolds satisfying the hard Lefschetz property.
\end{abstract}

2000 Mathematics Subject Classification: 14C30, 14F20, 14F25, 53D05.

Keywords and Phrases: Cohomology algebra, Hodge theory, hard Lefschetz theorem.

\section{Introduction}

We survey in this paper results obtained recently via the systematic study of cohomology algebras. All of our manifolds will be connected and orientable. Thus their cohomology algebras have 1-dimensional 0 and top degree term and satisfy Poincaré duality. We will be mainly interested in complex projective manifolds, compact Kähler manifolds, and compact symplectic manifolds.

Complex projective manifolds are complex submanifolds of some $\mathbb{C P}^{N}$. It is known that they are defined by homogeneous polynomial equations (Chow's theorem), so that they correspond to smooth complex projective varieties, (although it is in general crucial from the point of view of topology to distinguish between the abstract algebraic variety and the complex manifold, see [18], [7]).

*CNRS and IHÉS, E-mail: voisin@ihes.fr 
Kähler manifolds are complex manifolds admitting a Kähler metric, that is a Hermitian metric

$$
h=\sum_{i, j} h_{i j} d z_{i} d \bar{z}_{j}, h_{j i}=\overline{h_{i j}}
$$

for which the corresponding 2- form

$$
\omega=\frac{i}{2} \sum_{i, j} h_{i j} d z_{i} \wedge d \bar{z}_{j},
$$

which is a real form of type $(1,1)$ independent of the choice of local holomorphic coordinates $z_{1}, \ldots, z_{n}$, is closed : $d \omega=0$. The form $\omega$ is called the Kähler form of the metric.

As $\mathbb{C P}^{N}$ is Kähler, due to the presence of the Fubini-Study Kähler form, (which is the Chern form of the natural metric on the dual of the Hopf line bundle, induced by the standard Hermitian metric on $\mathbb{C}^{N+1}$ ), any complex projective manifold is Kähler.

The third kind of manifolds we will consider are symplectic compact manifolds, that is manifolds admitting a closed non degenerate 2-form. A Kähler manifold is in particular symplectic, because Kähler forms are symplectic forms. Notice that the set of Kähler forms is path-connected because it is a convex cone. So on a Kähler manifold there is in fact a well defined deformation class of symplectic forms, given by the set of Kähler forms.

Our results are of two kinds. The first one concerns the so-called Kodaira problem, which was solved negatively in [21]. We proved there that although compact Kähler manifolds are well known to be very restricted topologically, the projective complex manifolds are still more restricted topologically. More precisely, we show in that paper :

Theorem 1.1 Starting from dimension 4, there are compact Kähler manifolds which do not have the cohomology algebra of a complex projective manifold.

The second type of questions we shall consider is the following:

How restricted are the cohomology algebras of compact Kähler manifolds?

Note that this question is complementary to the formality theorem [8] which tells that the rational homotopy type of these manifolds (in the simply connected case) is determined by their rational cohomology algebra.

The only classical explicit constraints are the fact that the odd Betti numbers should be even, due to the Hodge decomposition (see subsection 1.1. below) and a stronger constraint due to the so-called hard Lefschetz property (see subsection 1.3.). 
Cohomology algebras in symplectic, Kähler and algebraic geometry 3

Examples of symplectic manifolds which are topologically non Kähler were constructed by Thurston [20], and McDuff [16] using the classical restriction on odd Betti numbers mentioned above. Later on, further examples were constructed by Bouyakoub [3], and Gompf [11] with even $b_{2 i+1}$ and thus satisfying the classical restriction, but not satisfying the hard Lefschetz property.

We shall construct here further examples of compact symplectic manifolds whith no odd degree cohomology, and satisfying the hard Lefschetz property, but not having the cohomology algebra of a compact Kähler manifold. In fact, all our examples are built starting from compact Kähler manifolds and performing standard operations like taking projective bundles on them or blowing-up symplectic submanifolds (see Theorem 3.1 and Proposition 3.4).

Our results show that there are many further constraints satisfied by the cohomology algebras of compact Kähler manifolds, deduced from the existence of a polarizable Hodge structure on their cohomology algebra (see subsection 1.2. below). Furthermore, we shall prove the following result (cf [23]), which also strongly restricts the cohomology algebra of topological direct summands of compact Kähler manifolds :

Theorem 1.2 Let $Z$ be a compact Kähler manifold, and assume $Z$ is homeomorphic to a product : $Z \cong X \times Y$, where $b_{1}(X)=0$. Then the cohomology algebras of $X$ and $Y$ carry polarizable Hodge structures inducing that of $Z$. Thus their cohomology algebras also satisfy the constraints mentioned above.

The rest of this section is devoted to the description of the main tools used: Hodge structures, polarization on them and compatibility with cup-product.

\subsection{Hodge structures}

The complex valued de Rham algebra $\mathcal{A}^{*}(X)$ of a complex manifold splits as a direct sum

$$
\mathcal{A}^{k}(X)=\bigoplus_{p+q=k} \mathcal{A}^{p, q}(X)
$$

where $\mathcal{A}^{p, q}(X)$ is the set of differential forms of type $(p, q)$. The Hodge decomposition theorem says that, when $X$ is compact Kähler, this decomposition descends to the cohomology groups with complex coefficients. Namely, defining

$$
H^{p, q}(X)=\frac{\{\text { closed forms of type }(p, q)\}}{\{\text { exact forms of type }(p, q)\}},
$$


one has for $X$ compact Kähler

$$
H^{k}(X, \mathbb{C})=\bigoplus_{p+q=k} H^{p, q}(X)
$$

From the definition above of $H^{p, q}(X)$, which is naturally a subspace of $H^{k}(X, \mathbb{C})$, it is obvious that

$$
\overline{H^{p, q}(X)}=H^{q, p}(X),
$$

where complex conjugation acts on $H^{k}(X, \mathbb{C})$. This property of the Hodge decomposition is called "Hodge symmetry". It has as an immediate consequence the following classical statement (which in the surface case characterizes Kähler surfaces among compact complex ones) :

Corollary 1.4. The odd Betti numbers $b_{2 i+1}(X)$ of a compact Kähler manifold are even.

Definition 1.5 A rational Hodge structure of weight $k$ is the data of a $\mathbb{Q}$-vector space $H$ and a decomposition

$$
H_{\mathbb{C}}:=H \otimes \mathbb{C}=\bigoplus_{p+q=k} H^{p, q}
$$

satisfying the Hodge symmetry property.

Taking for $H$ the cohomology with rational coefficients $H^{k}(X, \mathbb{Q})$, one gets a rational Hodge structure of weight $k$ on the degree $k$ cohomology of a compact Kähler manifold $X$.

There is another way to look at the Hodge decomposition. Namely, we can see it as the eigenspace decomposition for the following $\mathbb{C}^{*}$-action on $H_{\mathbb{C}}: z \in \mathbb{C}^{*}$ acts by multiplication by $z^{p} \bar{z}^{q}$ on $H^{p, q}$. The Hodge symmetry then simply says that $\mathbb{C}^{*}$ acts on $H_{\mathbb{R}}$.

\subsection{Hodge structures on cohomology algebras}

The main property of the Hodge decomposition that we shall exploit is the following :

Lemma 1.6 Let $X$ be compact Kähler and let

$$
\mu: H^{k}(X, \mathbb{C}) \otimes H^{l}(X, \mathbb{C}) \rightarrow H^{k+l}(X, \mathbb{C})
$$

be the cup-product map. Then

$$
\mu\left(H^{p, q}(X) \otimes H^{p^{\prime}, q^{\prime}}(X)\right) \subset H^{p+p^{\prime}, q+q^{\prime}}(X) .
$$

Proof. Indeed, the wedge product of a closed form of type $(p, q)$ and a closed form of type $\left(p^{\prime}, q^{\prime}\right)$ is a closed form of type $\left(p+p^{\prime}, q+q^{\prime}\right)$. 
Cohomology algebras in symplectic, Kähler and algebraic geometry 5

This tells us that the Hodge decompositions make the cohomology algebra $H^{*}(X, \mathbb{C})$ a bigraded algebra.

Another way to express this property is to say that the map $\mu$ is equivariant with respect to the $\mathbb{C}^{*}$-actions.

From this follows the following very useful lemma due to Deligne. Consider a cohomology algebra $A^{*}$ with rational coefficients. Assume there is a weight $k$ Hodge structure on each $A^{k}$ satisfying the compatibility condition (1.1). (We will then say that there is a Hodge structure on $A^{*}$.)

Let $Z \subset A_{\mathbb{C}}^{k}$ be a closed algebraic subset defined by homogeneous equations depending only on the product in $A^{*}$. (For example $Z:=\{a \in$ $\left.A_{\mathbb{C}}^{k}, a^{l}=0\right\}$ for a given $l$.) Let $Z^{\prime}$ be an irreducible component of $Z$, and let $\left\langle Z^{\prime}>\subset A_{\mathbb{C}}^{k}\right.$ be the complex vector subspace generated by $Z^{\prime}$. Lemma $1.7<Z^{\prime}>$ is stable under the Hodge decomposition of $A_{\mathbb{C}}^{k}$. Thus if $\left\langle Z^{\prime}\right\rangle$ is defined over $\mathbb{R}$, resp. $\mathbb{Q}$, that is

$$
<Z^{\prime}>=B \otimes \mathbb{C}
$$

for some $B \subset A_{\mathbb{R}}^{k}$ (resp. $B \subset A^{k}$ ), then $B$ is a real (resp. rational) sub-Hodge structure of $A_{\mathbb{R}}^{k}$, (resp. $\left.A^{k}\right)$.

Proof. Indeed, the definition of $Z$ and the equivariance of the product with respect to the $\mathbb{C}^{*}$-action show that $Z$ is stable under the $\mathbb{C}^{*}$-action. Thus $Z^{\prime}$ is also stable under this action, and so is $\left\langle Z^{\prime}\right\rangle$. But this is equivalent to saying that $\left\langle Z^{\prime}>\right.$ has an induced Hodge decomposition.

In practice, this lemma can be used to show that some even degree rational cohomology class $\alpha \in A^{2 i}$ must be a Hodge class (that is a rational class of type $(i, i)$ for any Hodge structure on the cohomology algebra $A^{*}$. It suffices for this to show that the line $\langle\alpha\rangle$ can be determined algebraically as above, because a Hodge structure of weight $2 i$ and rank 1 is necessarily of type $(i, i)$. In odd degree, one can apply the lemma to conclude that under the assumptions of Lemma 1.7, if $k$ is odd and $\left\langle Z^{\prime}\right\rangle$ is defined over $\mathbb{R}$, it must have even rank.

\subsection{Polarization}

Let $X$ be a compact Kähler manifold, and $\omega \in H^{2}(X, \mathbb{R})$ be a Kähler class. Then $(X, \omega)$ satisfies the hard Lefschetz property : for $n:=\operatorname{dim}_{\mathbb{C}} X$ and for any integer $k \leq n$, the cup-product by $\omega^{n-k}$

$$
\cup \omega^{n-k}: H^{k}(X, \mathbb{R}) \rightarrow H^{2 n-k}(X, \mathbb{R})
$$

is an isomorphism. Note that both sides have the same dimension by Poincaré duality. 
The hard Lefschetz property implies the Lefschetz decomposition (1.1) below (cf [24], 6.2.3): Let $\omega$ be a class satisfying the Lefschetz property above, and define for $k \leq n$ the primitive part $H^{k}(X, \mathbb{R})_{\text {prim }}$ by

$$
H^{k}(X, \mathbb{R})_{\text {prim }}:=\operatorname{Ker}\left(\cup \omega^{n+1-k}: H^{k}(X, \mathbb{R}) \rightarrow H^{2 n-k+2}(X, \mathbb{R})\right) .
$$

Then we have for $k \leq n$

$$
\bigoplus_{k-2 i \geq 0} H^{k-2 i}(X, \mathbb{R})_{\text {prim }} \stackrel{\sum_{i} \cup \omega^{i}}{\cong} H^{k}(X, \mathbb{R})
$$

Observe that the $\omega^{i} \cup H^{k-2 i}(X, \mathbb{R})_{\text {prim }}$ are real sub-Hodge structures of $H^{k}(X, \mathbb{R})$, which means that the corresponding complex vector spaces

$$
\omega^{i} \cup H^{k-2 i}(X, \mathbb{C})_{\text {prim }} \subset H^{k}(X, \mathbb{C})
$$

are stable under the Hodge decomposition. This follows from the fact that $\omega$ is of type $(1,1)$. If furthermore we can choose $\omega$ to be rational, then $H^{k}(X, \mathbb{R})_{\text {prim }}$ is in fact defined over $\mathbb{Q}$ and thus provides a subHodge structure of $H^{k}(X, \mathbb{Q})$.

To conclude, let us mention the Riemann bilinear relations, which will play an important role in the following sections.

$X, \omega$ being as above, we can construct for each $k \leq n$ a non degenerate Hermitian intersection pairing $h_{\omega}$ on $H^{k}(X, \mathbb{C})$, defined by :

$$
h_{\omega}(\alpha, \beta)=\iota^{k} \int_{X} \omega^{n-k} \cup \alpha \cup \bar{\beta}
$$

For bidegree reasons, we find that the Lefschetz and Hodge decomposition are orthogonal with respect to the pairing $h_{\omega}$.

Finally the second Riemann bilinear relations (see [24], Theorem 6.32) are restrictions on the signs of the Hermitian pairing $h_{\omega}$ restricted to the part

$$
H^{p, q}(X)_{\text {prim }} \subset H^{p, q}(X), p+q=k
$$

defined as $H^{p, q}(X)_{\text {prim }}=H^{p, q}(X) \cap H^{k}(X, \mathbb{C})_{\text {prim }}$.

Theorem 1.8 Let $X$ be a compact Kähler manifold with Kähler class $\omega$. Then the Hermitian form $h_{\omega}$ is definite of sign $(-1)^{\frac{k(k-1)}{2}} \iota^{p-q-k}$ on the component

$$
H^{p, q}(X, \mathbb{C})_{\text {prim }}, p+q=k
$$

of $H^{k}(X, \mathbb{C})$.

This is summarized by saying that the primitive pieces of the Lefschetz decomposition are real sub-Hodge structures, which are polarized by $\omega$. 
A point which will be crucial in section 2. is the following:

If $\omega$ can be chosen to be a rational cohomology class, then the pieces of the Lefschetz decomposition are rational sub-Hodge structures (namely, they are subspaces of $H^{k}(X, \mathbb{R})$ which are defined over $\mathbb{Q}$ ). Furthermore the Hermitian form $h_{\omega}$ as above is also rational in the sense that the corresponding bilinear form

$$
q_{\omega}(\alpha, \beta):=\int_{X} \omega^{n-k} \cup \alpha \cup \beta
$$

on $H^{k}(X, \mathbb{R})$ (which is symmetric for $k$ even and skew-symmetric for $k$ odd) is in fact rational on $H^{k}(X, \mathbb{Q})$.

We will speak in this case of rational Hodge structure which admits a rational polarization.

\section{The Kodaira problem}

Let $X$ be a Kähler compact manifold. Kodaira's embedding theorem says the following.

Theorem 2.1 (Kodaira, [13]) $X$ is projective if and only if $X$ carries a Kähler form whose cohomology class $\omega$ is rational, that is $\omega \in$ $H^{2}(X, \mathbb{Q})$.

Note that, in particular, if $X$ is Kähler and $H^{2,0}(X)=0$, then $X$ is projective. Indeed in that case $H_{\mathbb{R}}^{1,1}(X)=H^{2}(X, \mathbb{R})$, where $H_{\mathbb{R}}^{1,1}(X)$ is the set of cohomology classes which can be represented by a real closed form of type $(1,1)$. Since the cone of Kähler classes is open in $H_{\mathbb{R}}^{1,1}(X)$, it is then open in $H^{2}(X, \mathbb{R})$, and thus contains rational classes, since they are dense in $H^{2}(X, \mathbb{R})$.

Starting with a Kähler manifold $X$, one can deform the complex structure. It is known that the small deformations preserve the Kähler property and that the spaces $H^{p, q}$ vary differentiably inside the fixed space $H^{p+q}(X, \mathbb{C})$, which does not depend on the complex structure (see eg [24], 9.3.2). Given a family $\left(X_{t}\right)_{t \in B}$ of deformations of the complex structure on $X$, one can consider the set

$$
\cup_{t \in B} H_{\mathbb{R}}^{1,1}\left(X_{t}\right) \subset H^{2}(X, \mathbb{R})
$$

inside which sits as an open set the union of the Kähler cones $K_{t} \subset$ $H_{\mathbb{R}}^{1,1}\left(X_{t}\right)$. Assuming the union of the $K_{t}$ contains an open set of $H^{2}(X, \mathbb{R})$, then by the same density argument as above, it must contain a rational class, which means by Kodaira's theorem 2.1 that some $X_{t}$ is projective.

It turns out that this is precisely what happens in the case of Kähler surfaces.

Theorem 2.2 (Kodaira, [14]) A compact Kähler surface admits a (arbitrarily small) deformation which is projective. 
Kodaira's proof was obtained as a consequence of his classification of surfaces. A more direct proof, which consists in checking infinitesimally that the Kähler cones move with the complex structure so as to fill-in an open set, was given recently by Buchdahl [4], [5].

In higher dimension, the Kodaira theorem left open the question whether a compact Kähler manifold can be deformed to a projective one, a problem known as the Kodaira problem (see [9]), although it is not clear whether the question was asked by Kodaira himself.

Here we are considering more generally large deformations, that is, we say that $X$ is a deformation of $X^{\prime}$ if there exist connected analytic spaces

$$
\mathcal{X}, B
$$

a smooth proper holomorphic map

$$
\phi: \mathcal{X} \rightarrow B,
$$

and two points $t, t^{\prime} \in B$ such that $X_{t} \cong X, X_{t^{\prime}} \cong X^{\prime}$. Clearly, if $X$ and $X^{\prime}$ are deformations of each other, they are diffeomorphic, (although the diffeomorphism between them may not be canonically determined up to isotopy, because of the monodromy group of the fibration given by $\phi$ ). Indeed, this fibration can be trivialized way in a $C^{\infty}$ way over paths in $B$, and $B$ is path connected.

So, a fortiori, $X$ and $X^{\prime}$ are homeomorphic and in particular have the same homotopy type or cohomology algebra. Hence a weakening of the Kodaira problem asks the following :

Question. Does any compact Kähler manifold have the homotopy type of a projective complex manifold?

Note that there are no symplectic obstructions, by the work of either Gromov [12], Donaldson [10], Munoz et al [17], which show that any symplectic manifold can be realized as a symplectic submanifold of projective space.

We proved that the answer to this question is negative starting from dimension 4.

Theorem 2.3 (Voisin [21]) In any dimension $\geq 4$, there exist compact Kähler manifolds which do not have the homotopy type of complex projective manifolds.

In any dimension $\geq 6$ there exist simply connected such examples.

The method we sketch below to construct such examples plays on blowing-up certain complex tori along complex submanifolds, in such a way that the cohomology algebra of the blown-up manifold prevents it 
Cohomology algebras in symplectic, Kähler and algebraic geometry 9

to be projective. So these examples are in fact birationally equivalent to complex tori, which themselves deform to projective manifolds. However, later on in [22], we also proved the existence, starting from dimension 10, of compact Kähler manifolds, no birational model of which has the homotopy type of a complex projective manifold.

The examples constructed in [21] have the following shape (at least in the non simply-connected case). One considers complex tori $T$ admitting an endomorphism $\phi_{T}$. Later on, we will make an assumption on $\phi_{T}$, but for the moment we just assume that the eigenvalues of $\phi_{T *}$ acting on the tangent space of $T$ at 0 are all different from 0 or 1 .

It follows that inside $T \times T$ the four subtori

$$
\begin{gathered}
T_{1}:=T \times 0, T_{2}=0 \times T, \\
T_{3}=T_{\text {diag }}=\{(x, x), x \in T\}, T_{4}=T_{\text {graph }}=\left\{\left(x, \phi_{T}(x)\right), x \in T\right\}
\end{gathered}
$$

meet pairwise transversally at finitely many points.

We first blow-up the finitely many pairwise intersection points of these tori ; then the proper transforms $\widetilde{T}_{i}$ of the $T_{i}$ 's are smooth and do not meet anymore. So we can blow-up their union. The resulting compact complex manifold is Kähler because the Kähler property is stable under blow-ups.

We prove next that for adequate choice of $\left(T, \phi_{T}\right)$, the manifold $X$ so constructed does not have the homotopy type of a complex projective manifold. More precisely, let us make the following assumptions on $\left(T, \phi_{T}\right)$ :

$\left.{ }^{*}\right)$ the dimension $n$ of $T$ is $\geq 2$ and the endomorphism $\phi:=\phi_{T *}$ of $H_{1}(T, \mathbb{Q})$ satisfies the properties that all of its eigenvalues are distinct, and the Galois group of its characteristic polynomial acts as the symmetric group of $2 n$ elements on the set of eigenvalues.

The precise statement is then the following :

Theorem 2.4 Assume the assumptions (*). If $X^{\prime}$ is a Kähler compact manifold such that there exists a graded algebra isomorphism

$$
\gamma: H^{*}\left(X^{\prime}, \mathbb{Q}\right) \cong H^{*}(X, \mathbb{Q}),
$$

then $X^{\prime}$ is not projective.

The key point is the notion of polarized Hodge structure (see section 1.3.). Note that in degree 1 , the primitive cohomology $H^{1}(X, \mathbb{R})_{\text {prim }}$ is the full cohomology space $H^{1}(X, \mathbb{R})$.

The proof of Theorem 2.4 consists in showing that if we have $X^{\prime}$, $X$ and $\gamma$ as above, the Hodge structure on $H^{1}\left(X^{\prime}, \mathbb{Q}\right.$ ) (which has to be compatible via the cup-product with the Hodge structures on higher 
degree cohomology groups) cannot be polarized. Thus $X^{\prime}$ does not admit any rational Kähler class and is not projective.

The first step is the following:

Proposition 2.5 Let $L, L_{\mathbb{C}}=L^{1,0} \bigoplus \overline{L^{1,0}}$ be a weight 1 rational Hodge structure which admits an endomorphism $\psi$ (of Hodge structure) satisfying the Galois group assumption of $(*)$. Suppose that $r k L \geq 4$. Then this Hodge structure on $L$ cannot be polarized, that is, there is no element $\eta \in \bigwedge^{2} L^{*}$, such that

$$
\eta(\alpha, \beta)=0, i \eta(\alpha, \bar{\alpha})>0, \forall \alpha, \beta \in L^{1,0} .
$$

Proof. Indeed, the first condition tells that $\eta$ is of type $(1,1)$ for the induced weight 2 Hodge structure on $\bigwedge^{2} L^{*}$, while the second condition guarantees that $\eta \neq 0$. One looks then at the action $\wedge^{2} \psi^{*}$ induced by $\psi$ on $\bigwedge^{2} L^{*}$. The assumption on the Galois group then implies that this action is irreducible.

On the other hand, this action preserves the subspace $H d g^{2}\left(\bigwedge^{2} L^{*}\right)$, consisting of rational elements of $\bigwedge^{2} L^{*}$ which are also of type $(1,1)$. This subspace must then be either 0 or the whole of $\bigwedge^{2} L^{*}$. As $r k L \geq 4$, we have $\bigwedge^{2} L^{* 1,0} \neq 0$, so that $\bigwedge^{2} L^{*}$ is not completely of type $(1,1)$. Thus $H d g^{2}\left(\bigwedge^{2} L^{*}\right)=0$.

The second step will then be to show that if we have $X^{\prime}, X$ and $\gamma$ as above, there must be a splitting of the Hodge structure on $H^{1}\left(X^{\prime}, \mathbb{Q}\right)$

$$
H^{1}\left(X^{\prime}, \mathbb{Q}\right)=L \oplus L,
$$

and an automorphism $\psi$ of the sub-Hodge structure $L$ which satisfies the Galois group assumption of $(*)$.

More precisely, note that ${ }^{t} \phi$ acts on $H^{1}(T, \mathbb{Q})=H_{1}(T, \mathbb{Q})^{*}$, that this action satisfies the Galois group assumption of $(*)$, and that

$$
H^{1}(X, \mathbb{Q}) \cong H^{1}(T, \mathbb{Q}) \oplus H^{1}(T, \mathbb{Q}) .
$$

We now show the following proposition, which together with Proposition 2.5 completes the proof of Theorem 2.4 :

Proposition 2.6 Let $X^{\prime}, X$ and $\gamma$ as above. Then the induced decomposition

$$
H^{1}\left(X^{\prime}, \mathbb{Q}\right)=L \oplus L^{\prime},
$$

with

$$
L:=\gamma^{-1}\left(H^{1}(T, \mathbb{Q}) \times 0\right), L^{\prime}:=\gamma^{-1}\left(0 \times H^{1}(T, \mathbb{Q})\right)
$$

is a decomposition of $H^{1}\left(X^{\prime}, \mathbb{Q}\right)$ into two isomorphic sub-Hodge structures. Furthermore, the endomorphism ${ }^{t} \phi$ acting on $H^{1}(T, \mathbb{Q}) \cong L$ acts as an endomorphism of Hodge structure on $L$ (or $\left.L^{\prime}\right)$. 
Cohomology algebras in symplectic, Kähler and algebraic geometry 11

Proof. We use Deligne's lemma 1.7. Recall that $X$ was constructed from $T \times T$ by blowing-up complex submanifolds. Let us ignore the blowups of points, which is insignificant here, and consider the exceptional divisors $E_{1}, \ldots, E_{4}$ over the proper transforms $\widetilde{T}_{i}$. Their cohomology classes are rational cohomology classes $e_{i}$ on $X$, which are of type $(1,1)$ for the Hodge structure on $H^{2}(X, \mathbb{Q})$.

One then shows using Deligne's lemma that the $e_{i}^{\prime}:=\gamma^{-1}\left(e_{i}\right)$ are also of type $(1,1)$ for the Hodge structure on $H^{2}\left(X^{\prime}, \mathbb{Q}\right)$.

Finally, one notices that $H^{1}(T, \mathbb{Q}) \times 0 \subset H^{1}(X, \mathbb{Q})$ is recovered as $\operatorname{Ker} \cup e_{2}, 0 \times H^{1}(T, \mathbb{Q}) \subset H^{1}(X, \mathbb{Q})$ is recovered as $\operatorname{Ker} \cup e_{1}$, and that the antidiagonal of $H^{1}(T, \mathbb{Q})$ and the antigraph of ${ }^{t} \phi$ are recovered inside $H^{1}(T, \mathbb{Q}) \times H^{1}(T, \mathbb{Q})=H^{1}(X, \mathbb{Q})$ as $K e r e_{3}$ and Ker $e_{4}$ respectively.

It follows that one recovers similarly $L$ and $L^{\prime}$ as the kernels of $\cup e_{2}^{\prime}$ and $\cup e_{1}^{\prime}$ respectively, and as the $e_{i}^{\prime}$ are Hodge classes, it follows that $L$ and $L^{\prime}$ are sub-Hodge structures. Finally $\mathrm{Ker} \cup e_{3}^{\prime} \subset L \oplus L^{\prime}$ and Ker $\cup e_{4}^{\prime} \subset L \oplus L^{\prime}$ must be sub-Hodge structures of $L \oplus L^{\prime}=H^{1}\left(X^{\prime}, \mathbb{Q}\right)$. The first of them provides an isomorphism of Hodge structures $L \cong L^{\prime}$, while the second one shows that the endomorphism induced via $\gamma$ by ${ }^{t} \phi$ acts as an endomorphism of Hodge structures on $L$.

\section{Symplectic and Kähler manifolds}

A compact Kähler manifold $(X, \omega)$ can be seen, forgetting the complex structure, as a compact symplectic manifold. (Here we will not distinguish between the Kähler or symplectic form $\omega$ and its cohomology class.)

These symplectic manifolds are special because they satisfy the hard Lefschetz property (see section 1.3.). This implies among other things that their odd Betti numbers are even, because the isomorphism of cup-product with $\omega$

$$
\omega^{n-k}: H^{k}(X, \mathbb{R}) \rightarrow H^{2 n-k}(X, \mathbb{R}), 2 n=\operatorname{dim}_{\mathbb{R}} X
$$

is skew with respect to Poincaré duality.

In [23], we exhibit many more restrictions on the cohomology algebras of compact Kähler manifolds, leading to new examples of symplectic manifolds satisfying the hard Lefschetz property but topologically non Kähler. These restrictions come from the existence of polarizable Hodge structure on their cohomology algebras (see section 1.2. and 1.3.), that is a Hodge structure of weight $k$ on the degree $k$ cohomology, compatible with cup-product, and a real class of type $(1,1)$ which satisfies the hard Lefschetz property and the polarization condition of Theorem 1.8).

To give a sample of such results, we prove : 
Theorem 3.1 Let $X$ be a compact connected oriented manifold such that the algebra $H^{*}(X, \mathbb{Q})$ is generated in degree $\leq 2$. Let $E$ be a complex vector bundle on $X$, such that $c_{1}(E)=0$. Assume the cohomology algebra $H^{*}(\mathbb{P}(E), \mathbb{R})$ admits a Hodge structure. Then the cohomology algebra

$$
H^{*}(X, \mathbb{Q}) \subset H^{*}(\mathbb{P}(E), \mathbb{Q})
$$

has an induced Hodge structure, for which the Chern classes $c_{i}(E), i \geq 2$ are Hodge classes, that is, are of type $(i, i)$.

This leads to examples of compact symplectic manifolds satisfying the hard Lefschetz property, but topologically non Kähler, which are constructed as follows.

We start with a compact Kähler manifold $X$ together with a degree 4 rational cohomology class $\alpha$ which have the property that for no Hodge structure on $H^{*}(X, \mathbb{Q})$, the class $\alpha$ is of type $(2,2)$. The methods of the previous section easily lead to such examples.

Then we choose a complex vector bundle $E$ on $X$ which has $c_{1}(E)=$ $0, c_{2}(E)=N \alpha$ for some integer $N$. Theorem 3.1 above shows that the symplectic manifold $\mathbb{P}(E)$ does not have the cohomology algebra of a compact Kähler manifold.

Sketch of proof of Theorem 3.1. Notice that

$$
H^{1}(X, \mathbb{Q}) \cong H^{1}(\mathbb{P}(E), \mathbb{Q}) .
$$

As we know that the cohomology algebra of $X$ is generated in degree 2, in order to show that $H^{*}(X, \mathbb{Q}) \subset H^{*}(\mathbb{P}(E), \mathbb{Q})$ is a sub-Hodge structure, it suffices to prove it for the degree 2 part. Note that the real dimension of $X$ is even, $\operatorname{dim}_{\mathbb{R}} X=2 d$, because the real dimension of $\mathbb{P}(E)$ is even (the top degree cohomology group has rank 1 , hence its Hodge structure is of even weight). We claim that there exists $\beta \in H^{2}(X, \mathbb{Q})$ such that $\beta^{d} \neq 0$. This is clear because the dimension is even and the cohomology algebra is generated in degree $\leq 2$. It follows that we can recover algebraically

$$
H^{2}(X, \mathbb{C}) \subset H^{2}(\mathbb{P}(E), \mathbb{C})
$$

as a connected component of the set of $\alpha \in H^{2}(\mathbb{P}(E), \mathbb{C}), \alpha^{d+1}=0$. By Deligne's lemma 1.7, it follows that $H^{2}(X, \mathbb{Q})$ is a sub-Hodge structure of $H^{2}(\mathbb{P}(E), \mathbb{Q})$.

As for the statement that the Chern classes of $E$ are Hodge classes for this Hodge structures on $H^{*}(X, \mathbb{Q})$, we observe that by the definition of Chern classes, it suffices to show that

$$
\beta=c_{1}(\mathcal{H}) \in H^{2}(\mathbb{P}(E, \mathbb{Q})
$$

is a Hodge class for the given Hodge structure on $H^{2}(\mathbb{P}(E, \mathbb{Q})$, where $\mathcal{H}$ is the dual of the relative Hopf line bundle on $\mathbb{P}(E)$ ( $\operatorname{cf}[24], 11.2 .1$ ). 
Cohomology algebras in symplectic, Kähler and algebraic geometry 13

By Deligne's lemma 1.7, it suffices to give an algebraic recipe defining the line $\langle\beta\rangle$. Recalling that $c_{1}(E)=0$, one recovers $\langle\beta\rangle$ as a component of the set

$$
\left\{\alpha \in H^{2}\left(\mathbb{P}(E, \mathbb{C}), \tau_{*} \alpha^{r}=0 \text { in } H^{2}(X, \mathbb{C})\right\},\right.
$$

where $r=r k E$, and the Gysin map $\tau_{*}$ is recovered as the Poincaré dual of the pull-back map $\tau^{*}$ (we already know the inclusion $H^{*}(X, \mathbb{C}) \subset$ $\left.H^{*}(\mathbb{P}(E), \mathbb{C})\right)$. This concludes the proof.

In the paper [23], we exhibit further explicit restrictions on the cohomology algebras of compact Kähler manifolds, based on similar ideas. For example, the criterion that the odd Betti numbers should be even can be refined using Deligne's Lemma as follows:

Proposition 3.2 If a cohomology algebra $H^{*}(X, \mathbb{Q})$ admits a Hodge structure, then for each algebraic subset $Z^{\prime} \subset H^{2 k+1}(X, \mathbb{C})$ as in section 1.2., if the complex vector space $\left\langle Z^{\prime}>\subset H^{2 k+1}(X, \mathbb{C})\right.$ is defined over $\mathbb{R}$, it must be of even rank.

This is another source of construction of symplectic manifolds which are topologically non Kähler while satisfying the hard Lefschetz property.

The above results show that there are rather strong restrictions on the cohomology algebras of compact Kähler manifolds, deduced from the existence of polarizable Hodge structures on them. The following theorem shows that these topological restrictions are also inherited by their direct topological factors.

Theorem 3.3 Let $M^{*}$ be the cohomology algebra of a connected orientable manifold, which is equipped with a Hodge structure which can be polarized. Assume that

$$
M^{*} \cong A^{*} \otimes B^{*},
$$

where $A^{1}=0$. Then there are (polarizable) Hodge structures on $A^{*}$ and $B^{*}$ which induce the Hodge structure on $M^{*}$.

Theorem 1.2 of the introduction immediately follows, writing

$$
M^{*}=H^{*}(Z, \mathbb{Q}), A^{*}=H^{*}(X, \mathbb{Q}), B^{*}=H^{*}(Y, \mathbb{Q}) .
$$

Note that the assumption that $A^{1}=0$ (that is $b_{1}(X)=0$ in Theorem 1.2) is necessary, as shows the case of a one-dimensional complex torus $T \cong S^{1} \times S^{1}$.

Sketch of proof of Theorem 3.3. The key point is the fact that, as $A^{1}=0$, one has

$$
M^{2}=A^{2} \bigoplus B^{2} .
$$

We will content ourselves proving that $A^{2}$ and $B^{2}$ are sub-Hodge structures of $M^{2}$. Let $\omega \in M_{\mathbb{R}}^{2}$ be a polarizing class. If $2 n=\operatorname{dim}_{\mathbb{R}} M$, one 
has $\omega^{n} \neq 0$ in $M^{2 n}$. Writing $\omega=\alpha+\beta, \alpha \in A_{\mathbb{R}}, \beta \in B_{\mathbb{R}}$, one deduces from this that the dimensions of $A$ and $B$ should be even, say $2 a$ and $2 b$ respectively, and that

$$
\alpha^{a} \neq 0 \text { in } A^{2 a}, \beta^{b} \neq 0 \text { in } B^{2 b} .
$$

It then follows easily that

$$
A_{\mathbb{C}}^{2} \subset M_{\mathbb{C}}^{2}
$$

is an irreducible component of the set $\left\{m \in M_{\mathbb{C}}^{2}, m^{a+1}=0\right\}$. Deligne's lemma 1.7 then tells us that $A^{2} \subset M^{2}$ is a sub-Hodge structure. One argues similarly for $B^{2}$.

As a consequence of this, in the decomposition $\omega=\alpha+\beta$, one finds that $\alpha$ and $\beta$ are of type $(1,1)$ in $M^{2}$. Thus the fundamental classes (or top degree generators) $\alpha^{a}$ and $\beta^{b}$ of $A^{*}$ and $B^{*}$ respectively are also of type $(a, a)$ and $(b, b)$. One then proves by induction on $i$ that each $A^{i} \subset M^{i}, B^{i} \subset M^{i}$ is a sub-Hodge structure. This last step uses the Künneth decomposition, and the fact that the hard Lefschetz property is satisfied by $\alpha$ on $A^{*}$ and $\beta$ on $B^{*}$.

To conclude this section, we would like to show an example where the polarization conditions of Theorem 1.8 play a crucial role (actually, even in the previous section, we used only the hard Lefschetz property). In fact, when an oriented manifold $X$ has only even degree cohomology, one can always put the trivial Hodge structure on each $H^{2 i}(X)$, declaring that it is of type $(i, i)$. This trivially satisfies the compatibility conditions (1.1). We shall now construct a compact symplectic manifold satisfying the hard Lefschetz property, having only even degree cohomology, but not having the cohomology algebra of a compact Kähler manifold.

Let us consider a $K 3$ surface $S$. It is a compact Kähler surface with $b_{1}=0, b_{2}=22$ and the signature of the intersection on $H^{2}(S, \mathbb{R})$ is $(3,19)$.

Let us choose a basis $\alpha_{1}, \ldots, \alpha_{22}$ of $H^{2}(S, \mathbb{Q})$ consisting of classes close enough to a Kähler class. More precisely, we want that the $\alpha_{i}$ are classes of symplectic forms $\tilde{\alpha}_{i}$ close to a Kähler form, so that $\sum_{i} \tilde{\alpha}_{i}$ is still a symplectic form.

For an adequate choice of $M \in \mathbb{N}, M \alpha_{i}$ is an integral cohomology class for all $i$ 's. It then follows by Gromov embedding theorem [12], 3.4, that there exist symplectic embeddings

$$
\phi_{i}: S \hookrightarrow \mathbb{C P}^{N}
$$

such that $\phi_{i}^{*} \Omega=M \tilde{\alpha}_{i}$, where $\Omega$ is the Fubini-Study symplectic form on $\mathbb{C P}^{N}$. 
Cohomology algebras in symplectic, Kähler and algebraic geometry 15

Thus, the diagonal map

$$
\Phi=\left(\phi_{1}, \ldots, \phi_{22}\right): S \rightarrow\left(\mathbb{P}^{N}\right)^{22}
$$

is symplectic, because $\Phi^{*}\left(\sum_{i} p r_{i}^{*} \Omega\right)=M\left(\sum_{i} \tilde{\alpha}_{i}\right)$ is symplectic.

Let $X$ be the symplectic blow-up of $\left(\mathbb{P}^{N}\right)^{22}$ along $\Phi(S) . \quad X$ is a compact symplectic manifold with only even cohomology and satisfying the hard Lefschetz property. We conclude by proving:

Proposition $3.4 X$ does not have the cohomology algebra of a compact Kähler manifold.

Proof. As the map $\Phi^{*}$ is a surjection

$$
H^{*}\left(\left(\mathbb{P}^{N}\right)^{22}, \mathbb{Q}\right) \rightarrow H^{*}(S, \mathbb{Q})
$$

and the cohomology algebra of $\left(\mathbb{P}^{N}\right)^{22}$ is generated in degree 2 , it follows that the cohomology algebra of $X$ is generated in degree 2. Note that $H^{2}(X, \mathbb{Q})$ has for generators the classes $\omega_{i}$ of the differential forms $\tau^{*} p r_{i}^{*} \Omega$, where $\tau$ is the blow-down map, and the class $e$ of the exceptional divisor.

One checks easily that the lines $\left\langle\omega_{i}>\right.$ are irreducible components of the algebraic subset

$$
Z=\left\{a \in H^{2}(X, \mathbb{C}), a^{N+1}=0\right\} .
$$

By Deligne's lemma 1.7, it follows that for any compatible Hodge structure on $H^{*}(X, \mathbb{Q})$, the classes $\omega_{i}$ are of type $(1,1)$. As there is only one remaining generator $e$ of $H^{2}(X, \mathbb{Q})$, it must also be of type $(1,1)$ for such Hodge structure. As the cohomology algebra is generated in degree 2 , it follows that all the Hodge structures on $H^{2 i}(X, \mathbb{Q})$ should be trivial.

This contradicts now the possibility of having a polarizable Hodge structure on $H^{*}(X, \mathbb{Q})$. Indeed, as a consequence of Lefschetz decomposition and of Theorem 1.8, one has the Hodge index formula (see [24], 6.3.2), which gives the signature $\operatorname{sign}(Y)$ of the middle intersection form of a compact oriented manifold $Y$ of real dimension $4 n$, which admits a polarizable Hodge structure on its cohomology algebra:

$$
\operatorname{sign}(Y)=\sum_{a}(-1)^{a} h^{a, b}(Y) .
$$

In our case, we know that the Hodge numbers $h^{a, b}(X)$ should be 0 for $a \neq b$ and this equality would thus provide:

$$
\operatorname{sign}(X)= \pm \sum_{a} b_{2 a}(X) .
$$

(Here the sign \pm is due to the fact that the signature depends on the orientation. Here on the left we compute $\operatorname{sign}(X)$ for the natural orientation of $X$.) It is now easy to check that this formula is not satisfied. 


\section{References}

[1] J. Amorós, M. Burger, K. Corlette, D. Kotschick, D. Toledo. Fundamental groups of compact Kähler manifolds, Mathematical Surveys and Monographs, 44. American Mathematical Society, Providence, RI, 1996.

[2] I. Bauer, F. Catanese, F. Grunewald. The absolute Galois group acts faithfully on the connected components of the moduli space of surfaces of general type, preprint arXiv:0706.1466.

[3] A. Bouyakoub, Non existence de structures kaehlériennes sur les fibres principaux en tores, Rend. Sem. Fac. Sci. Univ. Cagliari 75 (2005), no. 1-2.

[4] N. Buchdahl. Algebraic deformations of compact Kähler surfaces, Math. Z. 253 (2006), no. 3, 453-459.

[5] N. Buchdahl. Algebraic deformations of compact Kähler surfaces II, to appear in Math. Zeitschrift, published online: 25 April 2007.

[6] G. Cavalcanti. The Lefschetz property, formality and blowing up in symplectic geometry, Trans. Amer. Math. 359 (2007), 333-348.

[7] F. Charles. Conjugate varieties with distinct real cohomology algebras, preprint arXiv:0706.3674.

[8] P. Deligne, Ph. Griffiths, J. Morgan, D. Sullivan. Real homotopy theory of Kähler manifolds, Inventiones Math. 2 (1975), 245-274.

[9] J.-P. Demailly, T. Eckl, T. Peternell. Line bundles on complex tori and a conjecture of Kodaira, Comment. Math. Helv. 80 (2005), no. 2, 229-242.

[10] S. Donaldson. Symplectic submanifolds and almost-complex geometry, J. Differential Geom. 44 (1996), no. 4, 666-705.

[11] R. Gompf. A new construction of symplectic manifolds, Ann. of Math. (2) 142 (1995), 527-595.

[12] M. Gromov. Partial Differential Relations, Ergebnisse der Mathematik und ihrer Grenzgebiete 3. Folge. Band 9, Springer-Verlag (1986).

[13] K. Kodaira. On Kähler varieties of restricted type (an intrinsic characterization of algebraic varieties), Ann. of Math. 60 (1954) 28-48.

[14] K. Kodaira. On compact complex analytic surfaces, I, Ann. of Math. 71 (1960), 111-152.

[15] E. Looijenga, V. Lunts. A Lie algebra attached to a projective variety, Invent. Math. 129 (1997), 361-412.

[16] D. McDuff. Examples of simply-connected symplectic nonKählerian manifolds, J. Differential Geom. 20 (1984), no. 1, 267277.

[17] V. Munoz, F. Presas, I. Sols. Almost holomorphic embeddings in Grassmannians with applications to singular symplectic submani- 
Cohomology algebras in symplectic, Kähler and algebraic geometry 17

folds. J. Reine Angew. Math. 547 (2002), 149-189

[18] J.-P. Serre. Exemples de variétés projectives conjuguées non homéomorphes, C. R. Acad. Sci. Paris 258 (1964), 4194-4196.

[19] C. Simpson. The construction problem in Kähler geometry. Different faces of geometry, 365-402, Int. Math. Ser. (N. Y.), 3, Kluwer/Plenum, New York, 2004.

[20] W. Thurston. Some simple examples of symplectic manifolds, Proc. Amer. Math. Soc. 55 (1976), no. 2, 467-468.

[21] C. Voisin. On the homotopy types of compact Kähler and complex projective manifolds, Inventiones Math. Volume 157, Number 2, (2004) $329-343$.

[22] C. Voisin. On the homotopy type of Kähler manifolds and the birational Kodaira problem, J. Differential Geom. 72 (2006), no. 1, $43-71$.

[23] C. Voisin. Hodge structures on cohomology algebras and geometry, preprint 2007, to appear in Math. Annalen.

[24] C. Voisin. Hodge Theory and Complex Algebraic Geometry, I, Cambridge studies in advanced mathematics 76, Cambridge Univ. Press (2002). 